\title{
Cloud monitoring system at Telescope Array site by Visible Fisheye CCD
}

\author{
Ryo Nakamura ${ }^{1, *}$, Takayuki Tomida ${ }^{1}$, Yasunori Saito ${ }^{1}$, and Katsuya Yamazaki $^{2}$ for the TA collaboration \\ ${ }^{1}$ Shinshu University, Nagano, Nagano, Japan \\ ${ }^{2}$ Kanagawa University, Yokohama, Kanagawa, Japan
}

\begin{abstract}
The Telescope Array (TA) is an international experiment studying ultra-high energy cosmic rays. TA uses the fluorescence detection technique to observe cosmic rays. In order to estimate the energy of cosmic rays with a fluorescence detector (FD), it is necessary to measure the condition of the observation area. Clouds affect the accuracy of the energy estimation. It is necessary to measure the existence of clouds and their direction in the Field Of View (FOV) of the FD. We developed a cloud monitoring system to measure the night sky weather using a CCD camera. In this report, we present our progress on our CCD cloud monitoring system.
\end{abstract}

\section{Introduction}

The Telescope Array (TA) experiment observes Ultra-high energy cosmic rays (UHECRs) with energy greater than $10^{18} \mathrm{eV}$ since 2008 in Utah, USA. The TA experiment is using two air shower detection techniques, the fluorescence detection technique and the particle detection technique to observe UHECRs[1][2]. We observe the secondary particles with a particle detector at the ground using scintillation counters.

We observe the fluorescence emission light generated by the interaction between secondary particles and atmospheric molecules using FDs. The TA experiment has 3 FD stations called Black Rock(BR), Long Ridge(LR) and Middle Drum(MD) as shown on figure 1. Each FD station has 12 or 14 telescopes. Cosmic rays are observed using FD at moonless and cloudless night. When there are clouds, the emitted fluorescence light is intercepted, scattered, and attenuated by them. In these cases we can't acquire correct data. Therefore it's very important to know the weather, especially the existence of clouds above the observation site. The TA experiment is using a night sky cloud monitoring system with visible CCD camera. This system is installed at 3 sites, the BR FD station, the LR FD station and the Central Laser Facility (CLF). The weather is monitored to know the respective weather. This monitoring system takes picture of the night sky every minute. These images are monitored by FD operators as one of factors to judge the conditions of operation. It is difficult for persons who aren't expert of the FD operations to judge the weather then the standard of the weather judgement is depending on observers.

We developed the cloud monitoring system to quantify the weather by a systematic evaluation. In this paper, we report our progress of development.

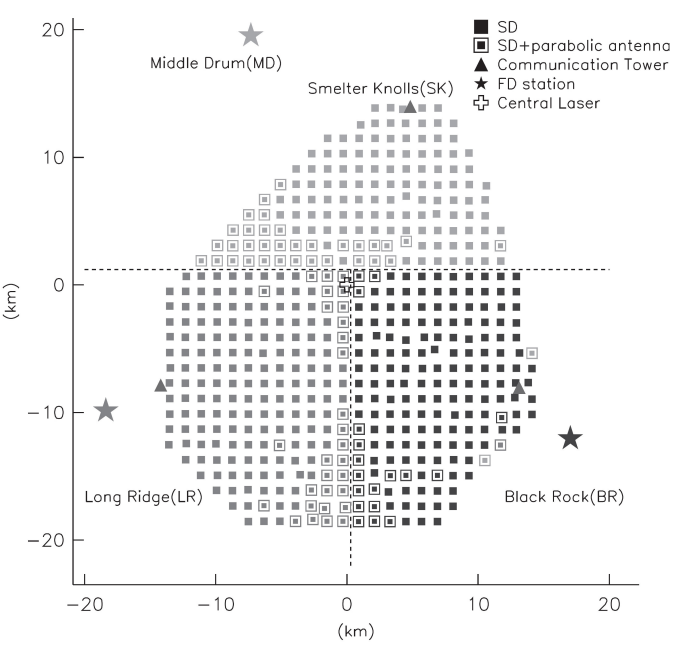

Figure 1: Detector map of TA experiment. Cloud monitoring system(CCD camera) is installed at 3 sites of BR, LR and CLF

\section{Cloud Monitoring System}

The cloud monitoring system consists of a visible light CCD camera with a fisheye lens, a housing dome and an image server. This system can be easily configured, it can be installed for less than $\$ 5,000$. The visible light CCD camera is a WAT $120 \mathrm{~N}+$ made by Watec, the fisheye lens is FE185C057HA-1 made by Fujinon. The fisheye lens can take a picture of the whole sky because it has a wide field of view of $185^{\circ} 0^{\prime} \times 154^{\circ} 1^{\prime}$. The housing dome is made of aluminum alloy, and the dome cover is made of plastic. It is possible to install and operate in outdoors all year. We use mmEye-S made by Brains as the image server. The generated image size is $720 \times 480$ pixels. It has a gray scale gradation of $0 \sim 255$ and it can recognize stars. The CCD camera exposes 8 seconds per minute.

*e-mail: 17w2064a@shinshu-u.ac.jp 
Figure 2 shows this overview of this system. A camera is installed on the roof of each building to improve the field of view.

The state of Utah where the TA experiment is located is very cold in winter, snow will offen persists for some time once it falls. When there is snow on the housing dome it may cut off the field of view. Therfore, we have installed a heater on the outside of the housing dome to melt snow in winter.

Figure 3 is the diagram of this system. The black solid lines are the power line. The black dashed line represent a signal line. Black double lines represents physical connection. The mmEye and CCD camera are connected via a $75 \Omega$ coaxial cable as a signal line. The output from CCD camera is sent to the mmEye directly. the mmEye is installed inside the building. The USB memory is connected to the mmEye(USB memory capacity is 256GB), and pictures are collected. In addition, an Ethernet cable is connected and accessed remotely from the network every minute, so we can see the picture while observing cosmic rays.

The CCD camera requires a $12 \mathrm{VDC}$ power supply, so that the DC power is supplied to the housing dome installed on the roof by an ACDC converter installed in the building. The height of the FD station is about $15 \mathrm{~m}$. In order to prevent a voltage drop, a DCDC converter (JCK2012S12 made by XP Power) is installed just before the CCD camera. The CCD camera generates heat in the body during operation. Noise is generated in the picture because the CCD camera is sensitive to heat. Therfore, we have installed two fans (RS Stock 668-8808 made by RS Pro) operating at 12 VDC.

Figure 4 shows the inside of the housing dome. The housing dome contains a CCD camera with a fisheye lens, the DCDC converter and two fans. One of the fans is installed directing air flow upward to prevent condensation, and the other is installed sideways to directly cool the CCD camera.

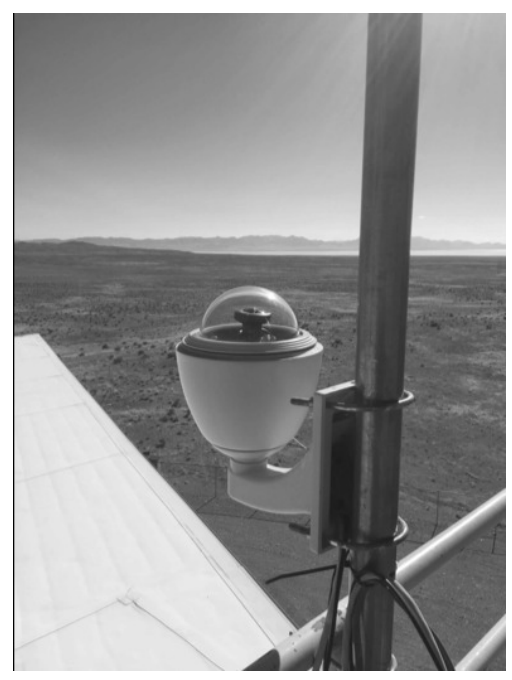

Figure 2: Overview of the Cloud Monitoring System. A camera is installed on a roof in each building to produce a good field of view of the night sky.

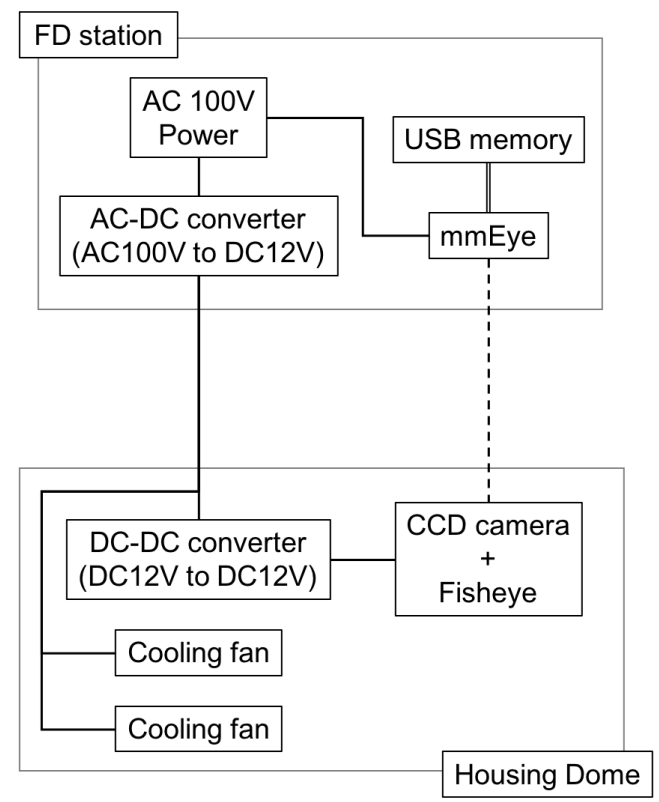

Figure 3: Diagram of the Cloud monitoring system. The black solid lines are power line. The black dashed line is signal line. Black double lines are physical connection.

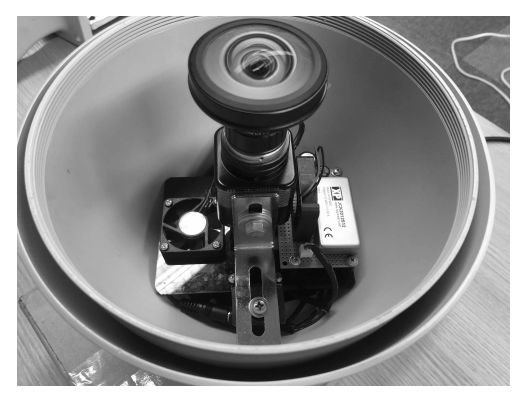

Figure 4: Inside of the housing dome. The housing dome consists of a CCD camera with fisheye lens, DCDC converter and two fans.

\section{Analysis}

Figure 5 shows examples of images taken by this system. This figure shows the change of the weather at that night. In a clear night sky image as shown in figure 5a, one may see many stars and the Milky Way. It looks bright overall. However, in an overcast image as shown in figure 5c, we can not see most of stars due to cloud cover. It looks dark overall. In partly overcast images as follow figure 5b, we can see stars within only a limited range. We consider that the weather can be evaluated by detecting stars from the image and counting the number of stars. We have developed a software to detect bright pixel clusters like stars from night sky images.

\subsection{Compare with Catalog Stats}

We compared detected stars with astronomical star a catalog to check the stars of the image in order to confirm 


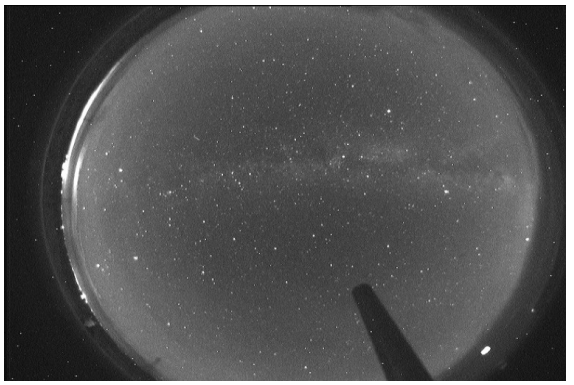

(a) Clear night at 02:09(UTC)

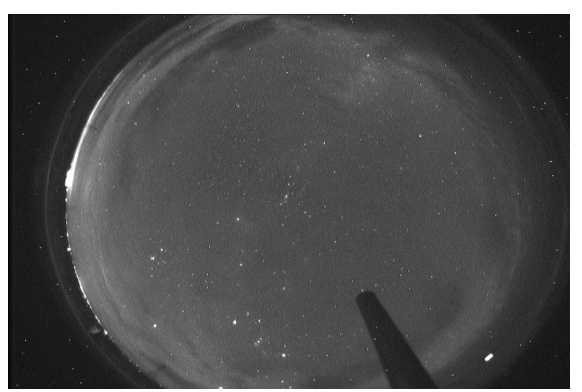

(b) Partly cloudy at 06:23(UTC)

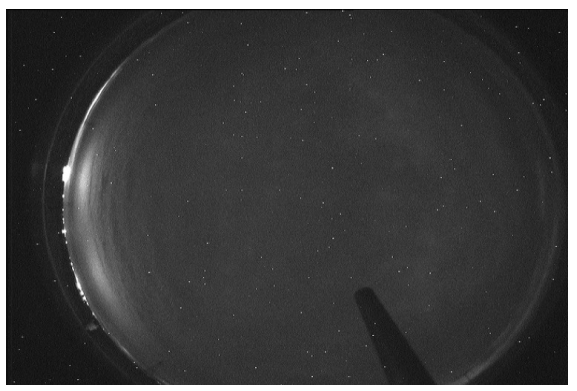

(c) Overcast at 11:00(UTC)

Figure 5: Example images from the Cloud Monitoring System from the taken on 20 Nov. 2014. Central Laser Facility.

that the detected stars are not noise or other artificial light. We use the Smithonian Astrophysical Observatory (SAO) Star Catalog. This catalog contains roughly 260,000 stars brighter than 9 magnitude, and this catalog is a widely used astronomical catalog. The SAO catalog includes the Right Ascension(R.A.) and Declination(Dec.). We converted R.A. and Dec. to CCD picture coordinates. We fitted with free parameters to overlay CCD pictures and the catalog stars as follow.

- Rotation from north. CCD camera is not installed heading at north.

- Scaling factor. Distortion is different in the vertical direction and the horizontal direction by fisheye.

- Shift in X-Y. The center of the image is not the zenith.

Figure $6 \mathrm{~b}$ shows the SAO star catalog position converted to CCD picture coordinates of figure 6a. The black circles are the SAO star catalog positions. The black dashed line represents the field of view of the CCD cam- era. In this analysis, we used the SAO star catalog brighter than 3.5 magnitude. Almost no star around the ground with the low angle of elevation can be seen due to scattering by aerosol, town lighting and distortion of the fisheye. Therefore, we only use stars with angles of elevation greater than $10^{\circ}$.

Figure 7 is the matching result. Gray solid circles are matched catalog stars. Black solid circles have no corresponding stars. We find corresponding stars from CCD pictures within accuracy of less than 2 degrees.

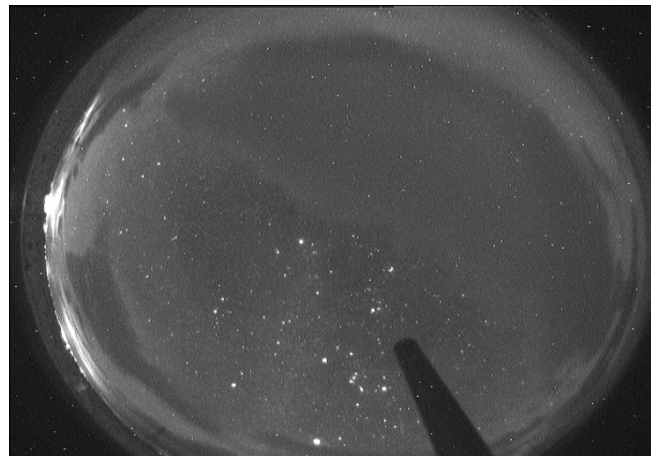

(a) Example of CCD picture.

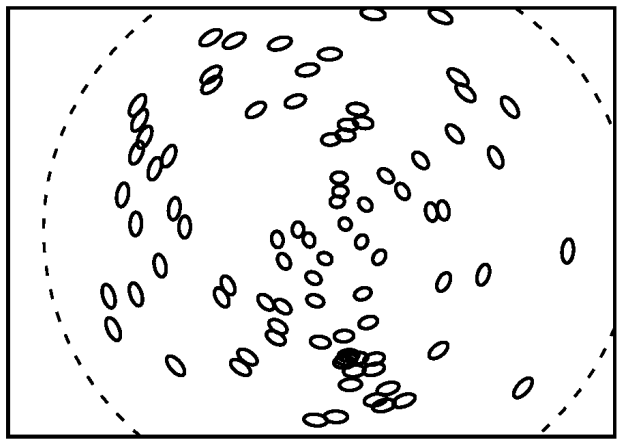

(b) SAO star catalog position converted to CCD picture coordinate of Fig. 6a. Black sold circles are SAO star catalog position. Black dashed circle is field of view of CCD camera.

Figure 6: Example of converted SAO star catalog position.

\subsection{Scoring Weather}

We can identify stars from the CCD picture by matching with the astronomical catalog. We evaluate the weather from number of stars which are detected in the CCD picture. We use the ratio of matched stars in each elevation angle for evaluating sky clearness as following equation 1 .

$$
\mathrm{R}=\frac{\mathrm{N}_{\mathrm{m}}}{\mathrm{N}_{\mathrm{e}}} \quad \begin{aligned}
& \mathrm{R}: \text { Clearness } \\
& \mathrm{N}_{\mathrm{m}}: \text { Num. of matched stars } \\
& \mathrm{N}_{\mathrm{e}}: \text { Num. of expected stars }
\end{aligned}
$$

The ratio of matched stars is calculated from the number of stars that corresponded to the number of stars ex- 


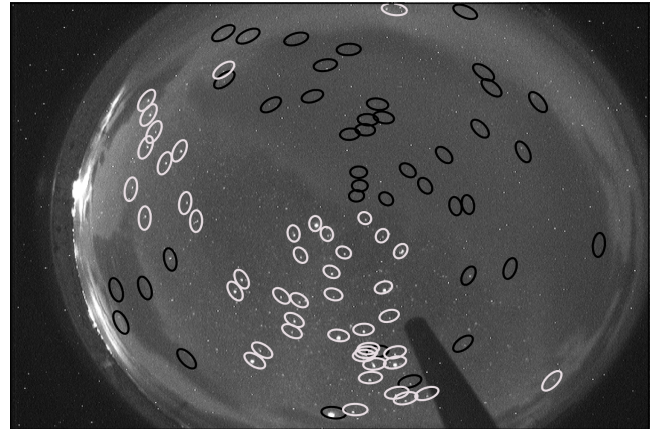

Figure 7: Result of the catalog star and CCD star matching. Gray solid circles are matched catalog stars. Black solid circles have no corresponding stars in the CCD picture due to cloud cover.

pected from CCD picture. For example, the number of expected stars in figure $6 a$ is 86 , and the number of matched stars is 46 , the ratio of matched stars is 0.53 .

We separated elevation angle every 30 degrees and separated azimuth angle in quarter as shown figure 8 . The numbers in each areas are ratios in its area. When the ratio is high, each area indicates in white means that the expectation is that the area is clear. When the ratio is low, each black area indicates cloudy sky.

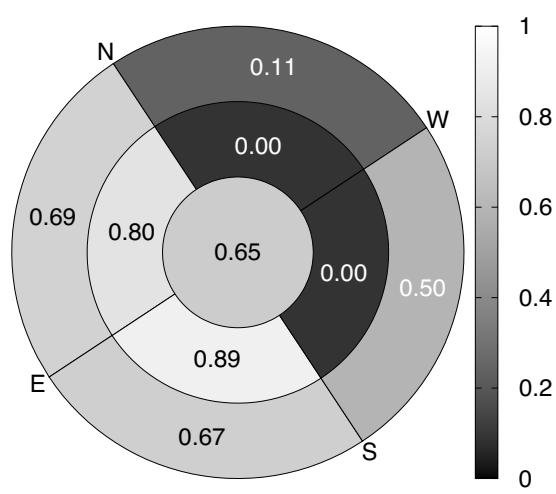

Figure 8: Result of scoring of Fig7. The number in each area are ratios in the area. When the ratio is high, it indicates clear viewing and it show as a light color. When the ratio is low, each area indicates cloudy viewing and it is a dark color.

\subsection{Time variation of weather}

Figure 9 shows the time variation of the ratio of matched stars over one day (Divided every 30 degrees.). The weather changes from cloudy to clear. Figure 10 shows the pictures picked up from each timing indicated by the gray dashed box in figure 9 (1) (3). (1) in figure 9 it is cloudy. (2) in figure 9 it is partly cloudy. (3) in figure 9 it is clear. We can see changing the weather as time passes and we can see that the weather and analysis results are corresponding as shown in figure 10 .

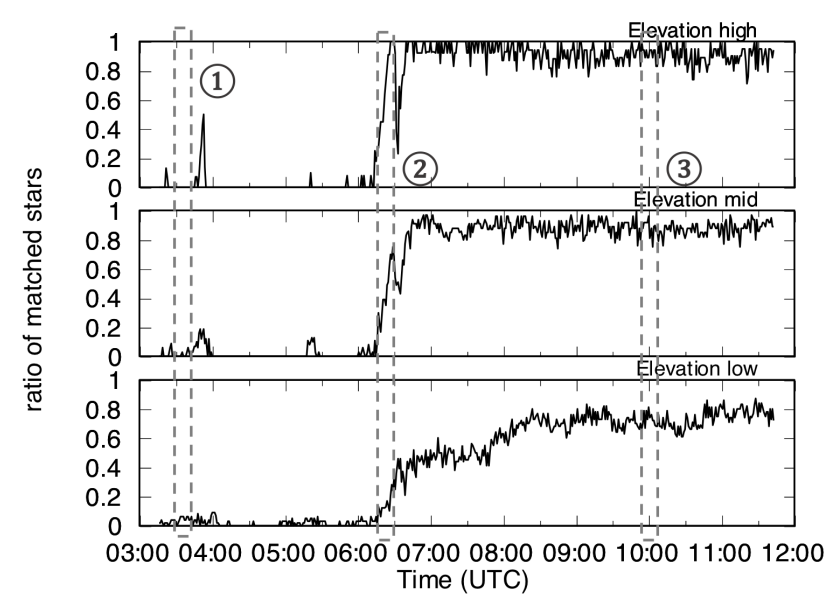

Figure 9: Time variation of ratio of matched stars in a day. (Bottom(Elevation low) : $0 \leq$ Elevation angle $\leq 30$, middle(Elevation mid) : $30<$ Elevation angle $\leq 60$, Top(Elevation high) : $60<$ Elevation angle $\leq 90)$ The weather changing from the cloudy to clear during observation.

Moreover, we can see less stars in the area of low elevation angle in spite of clear pictures. However, this is not a bad weather because the stars can not be seen due to scattering by atmospheric aerosols and twinkle star. The appearance of the star depends on the elevation angle. Therefore weather conditions are set for each elevation angle.

By taking a picture of CCD camera every 1 minute, we can know the weather in detail and with sufficient time resolution. Currently, the threshold of weather decision is a tentative value. The next step of our study is to decide standard value of sky clearness.

\section{Conclusion}

Our CCD cloud monitoring system, created at low cost with an off the shelf CCD camera, is taking pictures of the night sky including stars and is capable to find clouds. By comparing stars in the CCD picture with the SAO catalog, we can find corresponding stars from CCD pictures within accuracy a better than 2 degrees when using stars greater than 3.5 apparent magnitude. The ratio of matched stars was used for measuring the cloud existence. The formation of cloud can be found in detail by dividing area. (By Elevation angle and Azimuth angle.)

We are aiming to help the operation (Observation for ultra-high energy cosmic ray and other observation at night.) by using our cloud monitoring system. We are preparing a HTML information page (figure 11) to help observation, and that is expected to start being used in this winter at the Telescope Array observation.

\section{Acknowledgements}

The Telescope Array experiment is supported by the Japan Society for the Promotion of Science(JSPS) through Grants-inAid for Priority Area 431, for Specially Promoted Research 


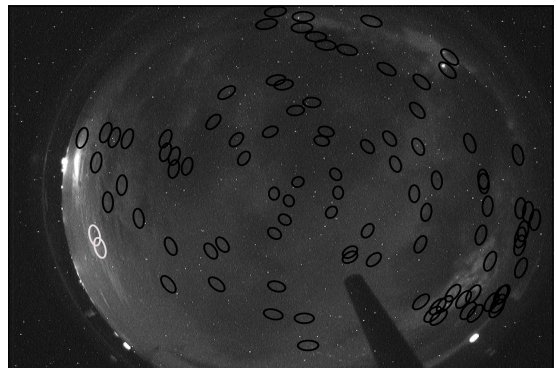

(a) Picture of (1) in Fig. 9(03:38(UTC)). Matched stars : 2 , Expected stars : 97.

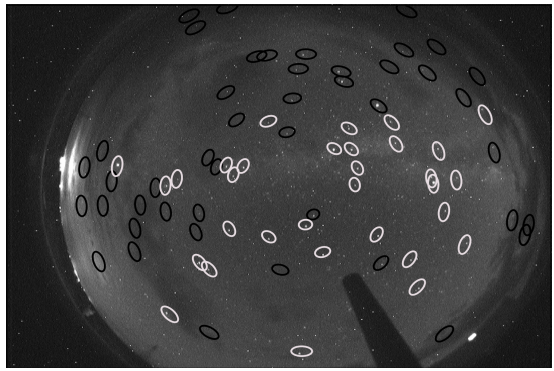

(b) Picture of (2) in Fig. 9(06:24(UTC)). Matched stars : 32 , Expected stars : 77.

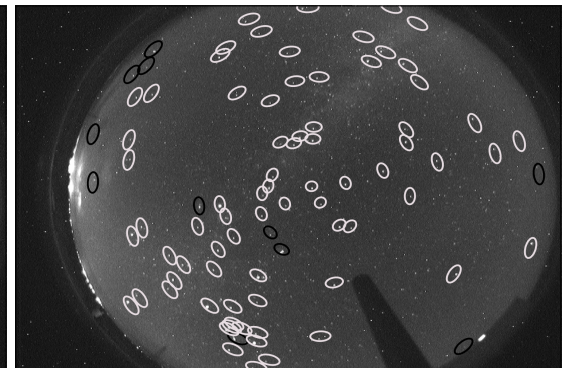

(c) Picture of (3) in Fig. 9(10:00(UTC)). Matched stars : 75 , Expected stars : 86.

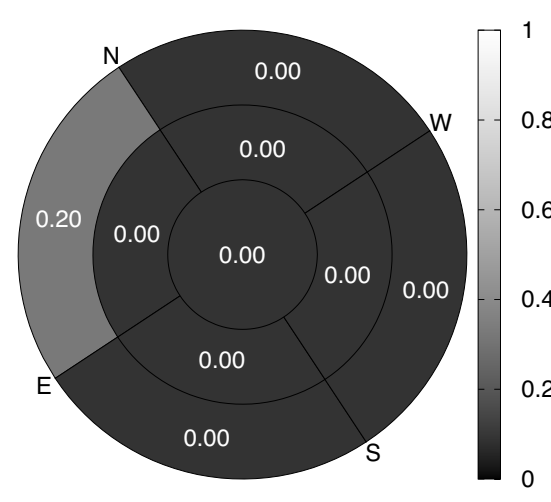

(d) Ratio of matched stars at Fig.10a

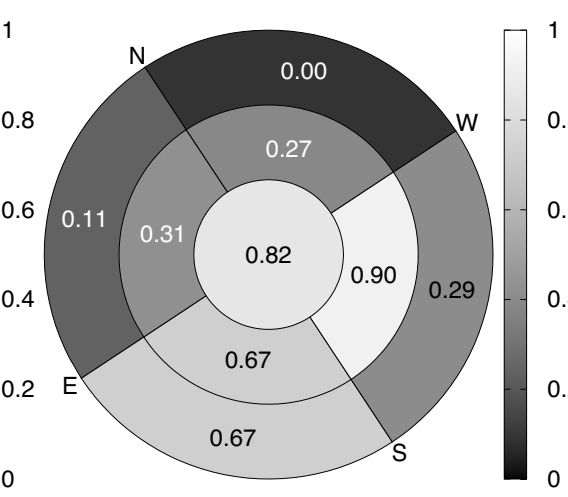

(e) Ratio of matched stars at Fig.10b

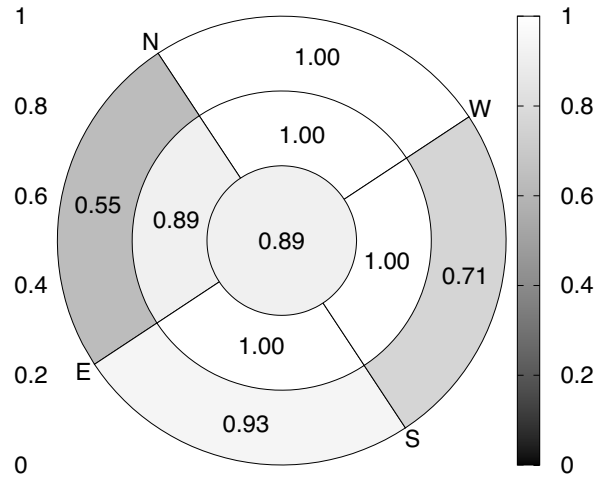

(f) Ratio of matched stars at Fig.10c

Figure 10: Pictures and its ratio of matched stars at the time indicated by gray dashed box (1) (3) $)$ in Fig. 9.

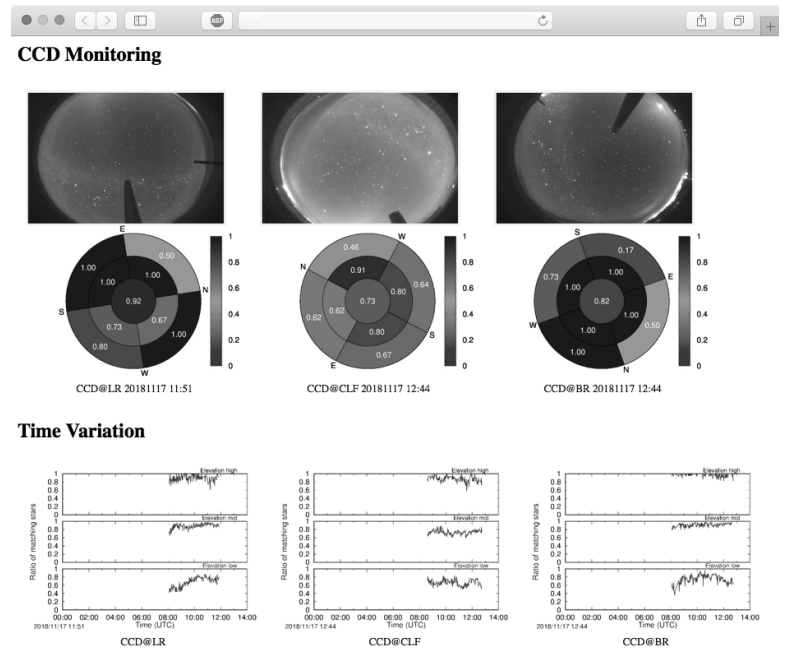

Figure 11: Example of HTML information page. This page update every minutes. Observer can be seen during FD observation.

JP21000002, for Scientific Research (S) JP19104006, for Specially Promoted Research JP15H05693, for Scientific Research (S) JP15H05741 and for Young Scientists (A) JPH26707011; by the joint research program of the Institute for Cosmic Ray Research (ICRR), The University of Tokyo; by the U.S. National Science Foundation awards PHY-0601915, PHY-1404495, PHY-1404502, and PHY-1607727; by the National Research Foundation of Korea (2016R1A2B4014967, 2016R1A5A1013277
, 2017K1A4A3015188, 2017R1A2A1A05071429) ; by the Russian Academy of Sciences, RFBR grant 16-02-00962a (INR), IISN project No. 4.4502.13, and Belgian Science Policy un-der IUAP VII/37 (ULB). The foundations of Dr. Ezekiel R. and Edna Wattis Dumke, Willard L. Eccles, and George S. and Dolores Doré Eccles all helped with generous donations. The State of Utah supported the project through its Economic Development Board, and the University of Utah through the Office of the Vice President for Research. The experimental site became available through the cooperation of the Utah School and Institutional Trust Lands Administration (SITLA), U.S. Bureau of Land Management (BLM), and the U.S. Air Force. We appreciate the assistance of the State of Utah and Fillmore offices of the BLM in crafting the Plan of Development for the site. Patrick Shea assisted the collaboration with valuable advice on a vari-ety of topics. The people and the officials of Millard County, Utah have been a source of steadfast and warm support for our work which we greatly appreciate. We are indebted to the Millard County Road Department for their efforts to maintain and clear the roads which get us to our sites. We gratefully acknowledge the contribution from the technical staffs of our home institutions. An allocation of computer time from the Center for High Performance Computing at the University of Utah is gratefully acknowledged.

\section{References}

[1] H.Tokuno et. al., NIM A, 676, 54-65(2012).

[2] T.Abu-Zayyad et. al., NIM A, 689, 87-97(2012). 